\title{
AFFECTIVE-SOUND EFFECTS ON DRIVING BEHAVIOUR
}

\author{
Jesús Serrano $^{1 \star}$, Leandro Luigi Di Stasi ${ }^{2 *}$, Alberto Megías ${ }^{3}$, Andrés Catena ${ }^{4}$ \\ 1,3,4 Learning, Emotion and Decision Group, Mind, Brain and Behavior Research Center, \\ University of Granada, Granada, Spain \\ ${ }^{2}$ Cognitive Ergonomics Group, Mind, Brain and Behavior Research Center, University of Granada, \\ Granada, Spain; Dept of Neurobiology, Barrow Neurological Institute, Phoenix, Arizona, USA
}

Submitted 31 August 2011; accepted 7 March 2012; first published online 25 October 2013

\begin{abstract}
Recent technological developments in active advanced driver assistance systems and in-car infotainment devices have contributed to reducing the number and severity of road accidents as well as improving and simplifying driver experience. However, these systems may impact driving performance in undesired ways, especially when emotionally-charged stimuli are used as warning signals. Emotional distraction can be a serious danger, causing delays in information processing, and reducing driving safety below minimal acceptable levels. Here we study the effect of emotionally-laden auditory signals on the speed of concurrent driving decisions. We distinguished two categories of behavioural responses: 'urgent' vs 'evaluative'. In the experiments reported here participants were quicker to evaluate whether a traffic scene was risky or not after hearing an emotionally-charged auditory stimulus than after a neutral one. However, urgent (braking) responses to the same scenes were not affected by the emotional quality of the auditory signal. Based on these results, we give preliminary advice on the design of guidelines for in-car interfaces particularly in the field of affective in-car computing.
\end{abstract}

Keywords: risk taking, risk perception, safety, traffic, emotion, ADAS.

Reference to this paper should be made as follows: Serrano, J.; Di Stasi, L. L.; Megías, A.; Catena, A. 2014. Affectivesound effects on driving behaviour, Transport 29(1): 100-106. http://dx.doi.org/10.3846/16484142.2013.815133

\section{Introduction}

How often have you been in a car with a child who started crying or laughing during the trip? What was your reaction? Could these events influence our response to emergencies or risky situations? Could they change our driving behaviour? Just as these emotionally-laden sounds may have an effect on the way you drive, the use of affective sounds as warning signals may have unexpected effects on the driver. This study investigated the effects of emotional sounds on the ability to evaluate and react to traffic situations.

A growing body of work has pointed out 'that emotion and cognition not only strongly interact in the brain, but that they are often integrated so that they jointly contribute to behaviour' (Pessoa 2008). It is, hence, likely that emotions also play a role in driving behavior (Groeger 2000); however there is no common agreement between international transport police organisations in considering an emotional distress as a road crash factor (NHTSA 2010). Particularly, in the field of road safety, there has been a surge of research on the effects of distractors (for example, a cell phone ringing) on driving quality (NHTSA 2009). However, less obvious sources of distractions (e.g. daydreaming or driving extremely emotionally aroused) have not been so frequently investigated (NHTSA 2009). Several countries (for example, New Zealand, Spain, or Canada), consider the emotional state of the driver in the more general category of inattention/distraction, which is how NHTSA started to classify emotion as from September 2010 (NHTSA 2010).

Driving under emotionally induced activation seems to cause an increase of unsafe on-road behaviour. Several researches have suggested that simple environmental noise (in a congestion situation, for example) or noise inside the passenger compartment of a car might increase the probability of aggressive driving behaviour (Mizell et al. 1997; Pullwitt 2008; Daimler Communications 2010) and reduce road safety. According to Groeger (2000), this can be due to the reorganization of goals

Corresponding author: Alberto Megías

E-mail: amegias@ugr.es

Copyright @ 2014 Vilnius Gediminas Technical University (VGTU) Press

http://www.tandfonline.com/TRAN 
and priorities under emotion and the restricted way in which the attention is deployed to the driving task. In this area, Briggs et al. (2011) demonstrated that driving under high-arousal due to negative emotions may give rise to goals that are irrelevant to driving and that compete with the relevant ones, reducing overall performance. However, the studies that indicated the relevance of emotion in traffic safety have been largely focused on emotional states characterized by extreme negative level of valence and high arousal, for example: provoked/frustrated (McGarva, Steiner 2000), angered (Mesken et al. 2007) or anxious (Briggs et al. 2011) drivers (see Table).

This fact could be related to the wrong belief that 'happy drivers are better drivers' (Eyben et al. 2010), see Fridman and Forster (2010), for a general review about the effects of affective experience on performance. Nevertheless the relationship between emotional states and risky driving behaviour seems to be more complex than it has been previously assumed - see, for example, Pêcher et al. (2009), Di Stasi et al. (2010a, b), Megías et al. (2013, 2011a, b). In general, these studies focused on the interaction between cognitive and emotional processes and the effects of these on risky behaviour, considering realistic experimental settings such as virtual driving simulators.

Table. Effects of driving emotionally aroused on the driving task. Strong emotions, both positive and negative, can affect drivers and how they use reduced risk driving decisions, adapted from OPI (2006)

\begin{tabular}{ll}
\hline Excited & drive inattentive \\
\hline Angry & drive aggressive \\
\hline Sad & slow reaction time \\
\hline Depressed & slow reaction time \\
\hline Anxious & slow reaction time \\
\hline Stressed & drive aggressive \\
\hline Worried & drive inattentive \\
\hline Frustrated & drive aggressive \\
\hline
\end{tabular}

In an original work, Pêcher et al. (2009) used music clips with different emotional valence (happy, sad and neutral) to influence driver's behaviour. 'Happy' music clips distracted drivers more significantly than sad and neutral clips, inducing them to decrease their speed and to impair lateral control (it has to be noted, that in this research confunding factors, as the rhythm and melody of the used song, could have influenced the effect valence on the measured dependent variables). Similar results were found by Di Stasi et al. (2010a). In this study the authors showed that emotional sounds could interfere with safe driving behaviour in a simulated road environment in which safety depended on the drivers ability to carry out a secondary detection task. Drivers' performance on the secondary task was influenced by the emotional features of the sound: a negative-valenced sound (scream) and a neutral abstract sound led to shorter reaction times than either a positive-valenced sound (laugh) or the absence of any sound when the complexity of the task was medium and high. Di Stasi et al. (2010b), using the same emotional sounds, studied the effects of an auditory warning system on the braking profile and gaze orientation in a number of presented risky scenarios. Some of the potential risky situations were cued by an emotional sound. Results showed that hearing a neutral abstract sound induced the riders to decrease their speed and focus their gaze on relevant areas of the visual field, while the emotional sounds (positive and negative) did not, decreasing road safety. Furthermore, Megías et al. (2011a, b), focusing on visual stimuli presentation, showed that emotional pictures (negative and positive) displayed while driving reduced the response times and increased the discriminability in subsequent risky situations.

All the above-cited research point out that 'to be emotionally aroused' could interfere with the on-going task performance more than neutral emotional states (Pêcher et al. 2009; Di Stasi et al. 2010b), and in addition, that negative stimuli appear to have the capacity to recruit attentional resources more readily (Di Stasi et al. 2010a; Megías et al. 2011b). Thus, these studies demonstrate that some kind of emotional stimuli can hinder the decision-making process while driving. Nevertheless most of this research on the role of emotions in the control of behaviour has focused on the manipulation of contextual (i.e. frame, see Slovic et al. 2004) and specific factors (i.e. moods, see Pessoa 2009). In fact, scarce attention has been devoted to uncover whether task features can modulate the effect of emotional state on driver behaviour in ecological road environments. In this vein, Megías et al. (2011a) distinguished between Urgent and Evaluative road user behaviours. Urgent behaviours are performed under high time-pressure and, when successful, they will help to avoid high negative outcomes (as when we brake because the vehicle ahead has stopped suddenly). On the other hand, Evaluative behaviours, are considered a type of categorization process (as for example to classify a road scene as risky or not). In addition, Megías et al. (2011a) suggested that Urgent and Evaluative behaviours are differentially affected by the emotional states.

\section{The Current Study}

Following the original works of Pêcher et al. (2009) and Di Stasi et al. (2010a, b) we investigated the effect of emotional and neutral auditory signals on the responses to risky traffic situations. Unlike Megías et al. (2011a, b), that focused on the influence of visual stimuli (emotionally charged pictures), here we wanted to study more ecological and commonly occurring traffic situations, i.e. to investigate the impact of emotionally-laden auditory stimuli on the driver's performance (response errors and speed). Two experiments were designed by changing the task requirements (see Method section for more details), that required different kinds of behaviour from the participant (namely: Urgent and Evaluative). Experiment 1 was designed to uncover how emotional sounds influence an urgent behaviour, and Experiment 2 was purported to demonstrate the emotional modulation of evaluative responses. 


\subsection{Experiment 1}

\subsubsection{Method and Participants}

A total of 19 undergraduate students (age: $M=21.37$, $S D=2.21$; age range: $18-27$ years; 3 men and 16 women) took part in this study in exchange for course credits. All had normal or corrected to normal vision and normal auditory capabilities. Furthermore, every participant had a driving license (having had their license for 18 months on average). Before the experiment they were informed of their rights according to the Helsinki declaration and they gave their informed consent.

\subsubsection{Apparatus and Materials}

Participants were seated comfortably in a darkened room, $57 \mathrm{~cm}$ from the screen centre $(1024 \times 728$ resolution), and held a mouse in front of them with both hands. A discriminative reaction time task was developed and controlled by the E-Prime software package.

A set of four naturalistic road pictures were presented to the participants. The pictures had been taken in a local highway (Granada, Spain) from behind the driver's seat, and showed a road with 4 different traffic conditions, two risky and two non-risky situations (see Fig. 1). For non-risky trials, pictures showed normal road situations (according to the Spanish road code); in contrast in the risky situations road traffic laws were violated (see also Serrano et al. 2011). Emotional warnings consisted of three auditory stimuli (presented at a level approximating $75 \mathrm{~dB}$ from fixed monitor speakers) that lasted for $850 \mathrm{~ms}$. The sounds were two emotionally charged voices: a baby's scream and a baby's laugh, selected from the International Affective Digitized Sounds - IADS - Bradley, Lang (1999) - numbers 261 and 110, respectively, see Di Stasi et al. (2010a, b), for further details, and a neutral sound: 'cork' (number 726, also selected from the IADS).

\subsubsection{Design and Procedure}

The experiment was arranged as a $3 \times 2$ repeated measures factorial design, with Emotional cue (Positive, Negative and Neutral) and Road Situation (Risky vs. NonRisky) as factors. The dependent variables were reaction time and accuracy for the risk decision-making task.

At the beginning of the experimental session, participants received a general description of the structure of the experiment and completed a demographic questionnaire assessing self-reported health, hearing ability, native language, and driving license status. During a short briefing, participants were informed about the timing of the experiment, and signed an informed consent form. The experiment consisted of 3 blocks of 144 trials, (24 trials of each experimental condition). It started after a training block (4 trials of each experimental condition), during which the experimenter checked that participants understood the task. Each trial follows the next sequence (Fig. 1): after a fixation point (750 ms), the emotional warning cue was presented for $850 \mathrm{~ms}$.

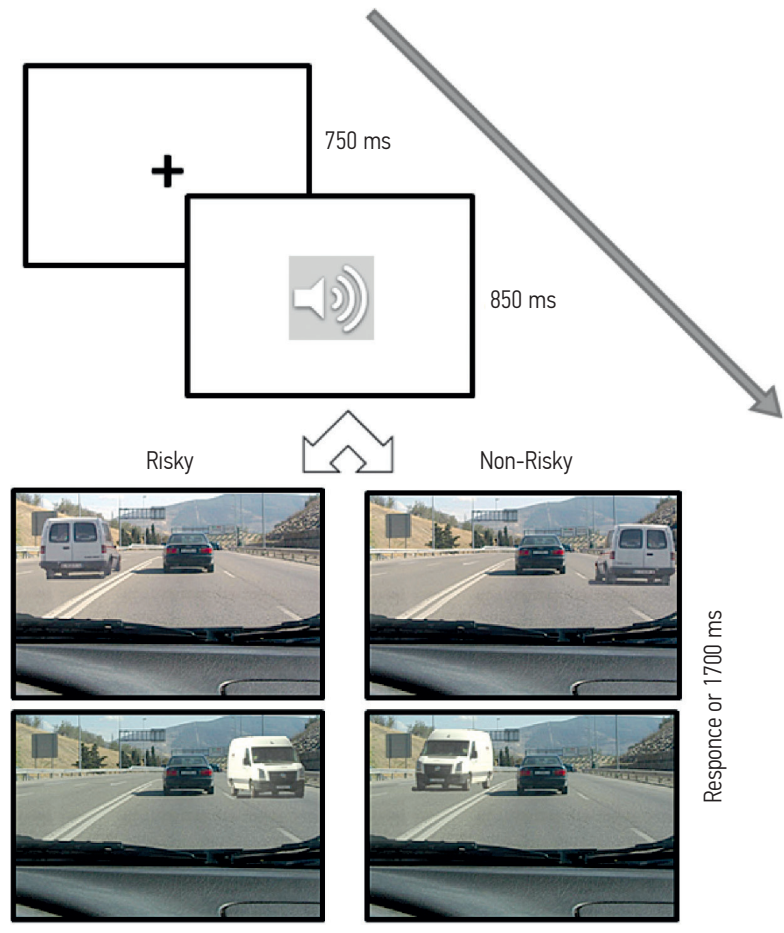

Fig. 1. Experimental procedure and parameters

Next, the target traffic picture (Risky or Non-Risky situation) was presented. Participants were asked to respond as quickly and accurately as possible, pressing mouse buttons, indicating whether they would break or not. The mapping between the right and the left mouse buttons and the type of response was balanced across participants. After the response or a maximum time of 1700 ms, a blank screen was shown for a variable interval between 500 to $1500 \mathrm{~ms}$. The sequence of trials was selected randomly for each participant. No feedback regarding the accuracy of their performance was given during the course of the experiment.

Following the recording session, participants were asked to rate their experienced valence and arousal, while viewing/hearing the sound cues and the target traffic scenes (4 road-pictures and the 3 emotional cues) using the paper-and-pencil version of the Self-Assessment-Manikin-Scales - SAM - (Lang 1980). To rate the level of valence, subjects were instructed to describe the stimuli 'pleasantness' as positive or negative on a 9-point rating scale (in which 1 point is the most negative and 9 points is the most positive). For arousal evaluation, participants had to describe the activation induced by the presented stimuli, from 'high activation' to 'low activation' on a nine-point rating scale (in which the point 1 represented the lowest induced activation and the point 9 represented the highest induced activation). By an analysis of the reliability of experimental data for both arousal and valence it has been estimated a consistency value of 0.86 and 0.87 respectively. The whole experimental session took about forty minutes. 


\subsubsection{Results}

Behavioural Data. Accuracy and reaction times (only for correct responses) were submitted to a 3 (Emotional cue: Positive, Negative, and Neutral) $\times 2$ (Road situation: Risky, Non-Risky) repeated measures analysis of variance (ANOVA). Previously, the data were checked to verify compliance with the assumptions of normality and homogeneity of variance for accuracy and reaction times (Kolmogorov-Smirnov test, $p>0.05$; Levene's test, $p>0.05)$. Results of the ANOVA on accuracy showed no significant main effects or interaction. According to the ANOVA on reaction times, there was a main effect of Road Situation, $F(1,18)=9.18, M S E=3553, p=0.007$ (Fig. 2). Reaction times were slower to Risky (797 ms) than to Non-Risky (763 ms) scenes. No other differences reached significance.

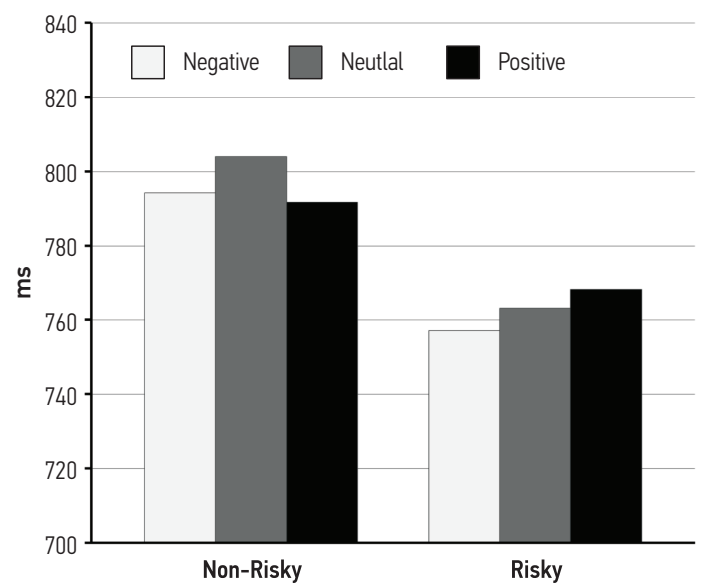

Fig. 2. Response latency (Experiment 1). Average reaction times according to risky situation and emotional warning cue

\subsubsection{SAM Rating}

Differences between valence and arousal of Risky and Non-Risky scenes were tested using a one-way analysis of variance on the SAM ratings for the pictures of those scenes. Risky scenes were rated less positive $(M=2.86$, $S D=1.07)$ than the Non-Risky ones $(M=5.42, S D=$ $1.29), F(1,18)=49.03, M S E=1.26, p<0.0001$. Consistent results were found for the analysis of induced-activation: Risky pictures generated higher activation $(M=$ $7.60, S D=1.11)$ than Non-Risky pictures $(M=5, S D=$ 1.24), $F(1,18)=37.26, M S E=1.73, p<0.0001$.

The level of activation varied across emotional cues, $F(2,36)=14.68, M S E=1.12, p<0.0001$. LSD post hoc analysis revealed that participants rated as more activating the positive cue $(M=5.89, S D=0.99)$ and the negative one $(M=6.57, S D=1.26)$ than the neutral one $(M=$ $4.73, S D=1.14)$. Also significant differences were found for the valence dimension, $F(2,36)=39.92, M S E=2.04$, $p<0.0001$. Valence was higher for positive $(M=7.73$, $S D=1.91)$ than for negative $(M=3.73, S D=1.52)$ and neutral sounds $(M=4.89, S D=1.24)$. The valence of the neutral cue also was higher than that of the positive cue.

\subsubsection{Discussion}

The main results of this study is that braking is faster, but equally accurate, for risky than for non-risky scenes, independently of whether the scene was preceded by emotional or neutral sounds. This result replicated the recent findings of Di Stasi et al. (2010a), that showed a reduction of driver reaction time when a negative valence was associated to the presented stimuli. Thus, the speed-up of the braking response can be accounted for by the emotional features of the target scene, so that high arousal/low valence seems to ease the behaviour. However, this effect can also be explained assuming that braking is an urgent response as not braking when the driving situation requires it can lead to fatal consequences (Megías et al. 2011a). Finally, the absence of an effect of the emotional cues can be the simple consequence of a failure in the selection of the emotional sounds. However, given that both valence and arousal induced by these stimuli were significantly different, it can be more parsimonious to assume that some kind of behaviours are harder to affect by emotional manipulations. We pursued further this idea en the next experiment, in which participants make an evaluation of the scene riskiness.

\subsection{Experiment 2}

\subsubsection{Methods and Participants}

A total of 20 undergraduate students (age: $M=21.7$, $S D=2.31$; age range: $18-27$ years; 4 men and 16 women) took part in this experiment in exchange for course credits. As in the first experiment, all participants possessed normal or corrected to normal visual and auditory capabilities and all had a driving license (having had their license for 20 months on average). They signed an informed consent form that described the risks of the study and the treatment of personal data. The experiment was conducted in accordance with the Helsinki Declaration.

\subsubsection{Apparatus and Design}

Apparatus and design used in this experiment were identical to those in Experiment 1, except for the question the participants were asked to respond to. In this case, the participants were requested to judge whether the traffic scene depicted was risky or not, using the same device as in Experiment 1. The response mapping was, again, counterbalanced across subjects.

\subsubsection{Results}

Behavioural Data. Accuracy and average reaction times for correct responses were submitted to a 3 (Emotional cue: Positive, Negative and Neutral) $\times 2$ (Road situation: Risky, Non-Risky) repeated measures analysis of variance. Previously, normality of data distribution (Kolmogorov-Smirnov test) and homogeneity of variances (Levene's test) were verified. Neither main nor interaction effects were significant for accuracy. Regarding reaction times, there were a significant main effect of emotional cue, $F(2,38)=5.74, M S E=579, p=0.007$ and $a$ 


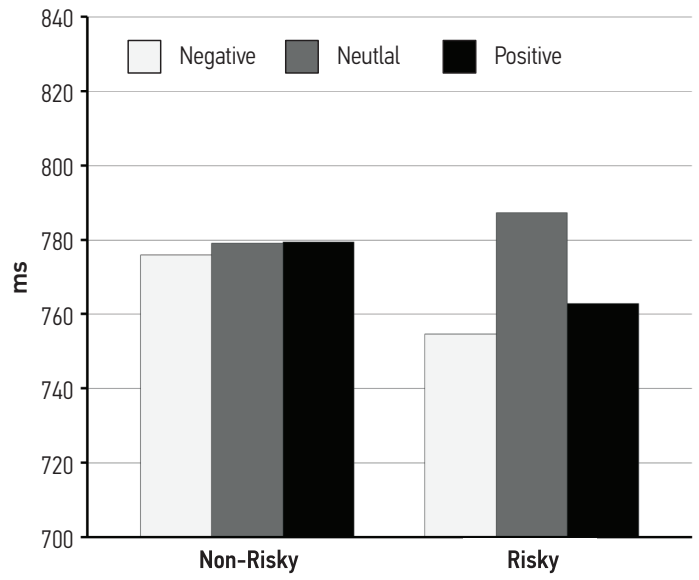

Fig. 3. Response latency (Experiment 2). Average reaction times according to risky situation and emotional warning cue

significant interaction effect (Road situation $\times$ emotional cue), $F(2,38)=4.06, M S E=615, p=0.003$ (Fig. 3). LSD post-hoc analysis of the interaction revealed that reaction times were shorter for negative cues $(755 \mathrm{~ms})$ and positive cues $(763 \mathrm{~ms})$ than for the neutral ones $(787$ $\mathrm{ms}$ ) only for the risky road situations.

\subsubsection{SAM Rating}

Differences between valence and arousal of Risky and Non-Risky scenes were tested using a one-way analysis of variance on the SAM ratings of those scenes. Risky situations were rated less positive $(M=2.5, S D=1.10)$ than the Non-Risky ones $(M=5.82, S D=1.18), F(1$, 19) $=80.57, M S E=1.37, p<0.0001$. Consistent results were found for the analysis of induced-activation: Risky situations generated higher activation $(M=7.17, S D=$ 1.44) than Non-Risky pictures $(M=4.02, S D=1.52)$, $F(1,19)=36.94, M S E=2.68, p<0.0001$.

Regarding the Emotional auditory cues, differences on arousal, $F(2,38)=4.99, M S E=3.01, p<0.025$, and valence, $F(2,38)=57.21, M S E=2.17, p<0.0001$, were observed. LSD post hoc analysis revealed that participants rated as more activating the positive cue $(M=$ $5.25, S D=1.85)$ and the negative one $(M=5.75, S D=$ $1.86)$ than the neutral one $(M=4.05, S D=1.50)$. Also significant differences were found for valence between positive, negative and neutral cues (positive warning cue: $M=7.95 ; S D=1.43$; negative warning cue: $M=$ 3.10; $S D=1.68$; neutral warning cue: $M=4.50 ; S D=$ 1.10).

\subsubsection{Discussion}

In stark contrast to Experiment 1, the emotional sounds affect speed of the evaluation of the scene's riskiness. As showed by Road situation $\times$ Emotional cue interaction, the emotionally charged stimuli (negative and positive) speed up the participant response in the case of risky situations. Given that these sounds have a significantly different valence, it seems that increased arousal induced by positive and negative sounds speeds up the evaluative response.

\section{General Discussions}

This study provides important results with regard to the influence that emotional stimuli may have on driver's behaviour. Taken together these findings suggest that affect-laden stimuli can modulate the driver's response differentially according to the specific task features, supplying additional data about the understanding of risk behaviour under emotional conditions (Megías et al. 2011a). Concretely, the emotional influence on driver behaviour becomes clear on the second experiment (when participants were asked to judge if road pictures were Risky vs. Non-Risky) if compared with the first one (to choose whether to brake or not). Our results provide additional evidence for supporting the importance of the task features in the study of driving behaviour and add valuable information on the influence of affective sounds in road situations.

\section{Conclusions}

Summarizing all aspects discussed in this report, it becomes clear that emotions could be a key issue in the future road-safety and in-car driving support devices. Since the first investigations (e.g. Doob, Gross 1968) there is still little available research on the emotional aspects of Driver-Vehicle-Environment interaction and how this impacts the driving experience (Gomez et al. 2008). Just recently there has been an increased interest by automotive industry in more intelligent and emotional car interfaces, progressively integrating more technologies into modern vehicles (Andrews 2001). The main challenges for next generation cars are closely related to the design and development of a set of in-vehicle affective systems able to increase road safety (Eyben et al. 2010). From a practical point of view our results could be considered as the first step to the development of an emotionally responsive car (Jones, Jonsson 2008). Our results show that emotional cues preceding the target scene modulate only evaluative behaviours. Considering that generally driving (as well as our daily experience) is facing with this specific kind of behaviour (Megías et al. 2011a, b), our data could make a valuable contribution to improve road safety, by designing an in-car driver support tool to counter-steer extreme emotional valence/arousal states. To provide an example, an extreme euphoric driver could be led to a more neutral emotional state by playing some relaxing music or showing some low valance/arousal pictures.

There is another fundamental contribution that our findings provide to the hazard perception research (Groeger 2000): the effect of task features on driver behaviour. Our data shows that driver response, when using a key-press responses in a YES-NO procedure, depends crucially on the type of instructions given to the participants. In the experiments reported here, different results were obtained, just by changing the task features. However, more research is needed to confirm this result.

To conclude, it is our hope that this work spurs further research on the effects of realistic affective stimuli 
on complex and ecological settings, and encourages the use of basic research in the service of the design of incar affective computing (Eyben et al. 2010; Trick et al. 2012, Megías et al. 2012).

\section{Acknowledgements}

This work was financed by Junta de Andalucía (Spain) PB06-02375 grant to A. Catena, Spanish MICINN PSI2009-12217 grant to A Maldonado, Junta de Andalucía (Spain) PB09-SEJ4752 grant to A. Cándido, and by the Spanish Ministry of Economy and Finance (Project PSI2012-39292).

L. L. Di Stasi was supported by the MEC-Fulbright Postdoctoral Fellowship Program (Grant PS-2010-0667).

We would like to thank D. Contreras for his enlightening discussion on the experimental results and for language revisions, and Prof. R. Garcia-Retamero for help in taking pictures.

\section{References}

Andrews, P. 2001. Affective Computing: Knowing how you Feel. Executive Tek Report, IBM Corporation, G510-1638-00. 4 p. Available from Internet: http://www-935.ibm.com/ services/fr/igs/pdf/affective-computing-knowing-how-youfeel.pdf

Bradley, M. M.; Lang, P. J. 1999. International affective digitized sounds (IADS): stimuli, instruction manual and affective ratings. Technical Report No. B-2. The Center for Research in Psychophysiology, University of Florida, Gainesville, FL.

Briggs, G. F.; Hole, G. J.; Land, M. F. 2011. Emotionally involving telephone conversations lead to driver error and visual tunnelling, Transportation Research Part F: Traffic Psychology and Behaviour 14(4): 313-323. http://dx.doi.org/10.1016/j.trf.2011.02.004

Daimler Communications. 2010. 125 Years of Innovation. 141 p. Available from Internet: http://www.daimler.com/ Projects/c2c/channel/documents/1960758_PM_125_ Jahre_Innovation_en.pdf

Di Stasi, L. L.; Contreras, D.; Cañas, J. J.; Cándido, A.; Maldonado, A.; Catena, A. 2010a. The consequences of unexpected emotional sounds on driving behaviour in risky situations, Safety Science 48(10): 1463-1468. http://dx.doi.org/10.1016/j.ssci.2010.07.006

Di Stasi, L. L.; Renner, R.; Staehr, P.; Helmert, J. R.; Velichkovsky, B. M.; Cañas, J. J.; Catena, A.; Pannasch, S. 2010b. Saccadic peak velocity sensitivity to variations in mental workload, Aviation, Space, and Environmental Medicine 81(4): 413-417. http://dx.doi.org/10.3357/ASEM.2579.2010

Doob, A. N.; Gross, A. E. 1968. Status of frustrator as an inhibitor of horn-honking responses, Journal of Social Psychology 76(2): 213-218.

http://dx.doi.org/10.1080/00224545.1968.9933615

Eyben, F.; Wöllmer, M.; Poitschke,T.; Schuller, B.; Blaschke, C.; Färber, B.; Nguyen-Thien, N. 2010. Emotion on the roadnecessity, acceptance, and feasibility of affective computing in the car, Advances in Human-Computer Interaction 2010: 1-17. http://dx.doi.org/10.1155/2010/263593

Friedman, R. S; Förster, J. 2010. Implicit affective cues and attentional tuning: an integrative review, Psychological Bulletin 136(5): 875-893. http://dx.doi.org/10.1037/a0020495
Gomez, R. F.; Popovic, V.; Bucolo, S. 2008. Emotional driving experiences, in Desmet, P.; Van Erp, J.; Karlsson, M. A. (Eds.). Design and Emotion Moves. Cambridge Scholars Publishing, Newcastle upon Tyne, 141-164.

Groeger, J. A. 2000. Understanding Driving: Applying Cognitive Psychology to a Complex Everyday Task. Routledge. 272 p.

Jones, C. M.; Jonsson, I.-M. 2008. Using paralinguistic cues in speech to recognise emotions in older car drivers, Lecture Notes in Computer Science 4868: 229-240.

http://dx.doi.org/10.1007/978-3-540-85099-1_20

Lang, P. J. 1980. Behavioral treatment and bio-behavioral assessment: computer applications, in Sidowsky, J. B.; Johnson, J. H.; Williams, T. A. (Eds.). Technology in Mental Health and Care Delivery Systems, Norwood, 119-137.

McGarva, A. D.; Steiner, M. 2000. Provoked driver aggression and status: a field study, Transportation Research Part F: Traffic Psychology and Behaviour 3(3): 167-179. http://dx.doi.org/10.1016/S1369-8478(00)00023-1

Megías, A.; Di Stasi, L. L.; Maldonado, A.; Catena, A.; Cándido, A. 2013) Emotion-laden stimuli influence our reactions to traffic lights, Transportation research part F: Traffic Psychology and Behaviour. http://dx.doi.org/10.1016/j.trf.2013.09.017

Megías, A.; Maldonado, A.; Catena, A.; Cándido, A. 2012. Two ways of understanding the emotional influence in traffic psychology, Securitas Vialis 4(3): 143-152. http://dx.doi.org/10.1007/s12615-012-9064-x

Megías, A.; Maldonado, A.; Cándido, A.; Catena, A. 2011a. Emotional modulation of urgent and evaluative behaviors in risky driving scenarios, Accident Analysis and Prevention 43(3): 813-817. http://dx.doi.org/10.1016/j.aap.2010.10.029

Megías, A.; Maldonado, A.; Catena, A.; Di Stasi, L. L.; Serrano, J.; Cándido, A. 2011b. Modulation of attention and urgent decisions by affect-laden roadside advertisement in risky driving scenarios, Safety Science 49(10): 1388-1393. http://dx.doi.org/10.1016/j.ssci.2011.06.001

Mesken, J.; Hagenzieker, M. P.; Rothengatter, T.; De Waard, D. 2007. Frequency, determinants, and consequences of different drivers' emotions: an on-the-road study using selfreports, (observed) behaviour, and physiology, Transportation Research Part F: Traffic Psychology and Behaviour 10(6): 458-475. http://dx.doi.org/10.1016/j.trf.2007.05.001

Mizell, L.; Joint, M.; Connell, D. 1997. Aggressive Driving: Three Studies. 41 p. Available from Internet: https://www. aaafoundation.org/sites/default/files/agdr3study.pdf

NHTSA. 2009. An Examination of Driver Distraction as Recorded in NHTSA Databases (DOT HS 811 216).Washington, DC: NHTSA [National Highway Traffic Safety Administration].

NHTSA. 2010. Overview of results from the international traffic safety data and analysis group survey on distracted driving data collection and reporting as recorded in NHTSA databases (DOT HS 811 404). Washington, DC: NHTSA [National Highway Traffic Safety Administration].

OPI. 2006. Effect of Emotions and Disabilities on the Driving Task. M17 Emotions-Disabilities-1. The Montana Office of Public Instruction. 18 p. Available from Internet: http:// opi.mt.gov

Pêcher, C., Lemercier, C.; Cellier, J.-M. 2009. Emotions drive attention: effects on driver's behaviour, Safety Science 47(9): 1254-1259. http://dx.doi.org/10.1016/j.ssci.2009.03.011

Pessoa, L. 2008. On the relationship between emotion and cognition, Nature Reviews Neuroscience 9: 148-158. http://dx.doi.org/10.1038/nrn2317 
Pessoa, L. 2009. How do emotion and motivation direct executive control?, Trends in Cognitive Sciences 13(4): 160-166. http://dx.doi.org/10.1016/j.tics.2009.01.006

Pullwitt, E. 2008. Driver behaviour and noise, in SILENCE Final Training Conference (Prezentation), 28-29 May, 2008, Bergisch-Gladbach, Germany. Available from Internet: http://www.silence-ip.org

Serrano, J.; Di Stasi, L. L.; Megías, A.; Catena, A. 2011. Effect of directional speech warnings on road hazard detection, Traffic Injury Prevention 12(6): 630-635. http://dx.doi.org/10.1080/15389588.2011.620661

Slovic, P.; Finucane, M. L.; Peters, E.; MacGregor, D. G. 2004. Risk as analysis and risk as feelings: some thoughts about affect, reason, risk, and rationality, Risk Analysis 24(2): 311322. http://dx.doi.org/10.1111/j.0272-4332.2004.00433.x

Trick, L. M.; Brandigampola, S.; Enns, J. T. 2012. How fleeting emotions affect hazard perception and steering while driving: the impact of image arousal and valence, Accident Analysis and Prevention 45: 222-229.

http://dx.doi.org/10.1016/j.aap.2011.07.006 